\title{
Teledermatology for a case of morphoea
}

John O’Bryen, James Muir

CASE

A woman aged 40 years presented to her general practitioner (GP) with a changing plaque on her left breast. She stated that it had appeared over the prior year and was mildly itchy, and she thought it had been triggered by her toddler scratching the location during breastfeeding. Three months ago she initiated a course of clotrimazole $1 \%$ cream with no effect. Her background included Hashimoto's thyroiditis and vulval lichen sclerosus, for which she received annual vulvoscopy by a gynaecologist. On examination, there was a circumscribed $3 \times 3 \mathrm{~cm}$ indurated, hypopigmented plaque on her left lateral breast (Figure 1). There was lack of hair, and it had a wrinkled appearance. Examination of her breasts, skin, hair and nails was otherwise unremarkable.

\section{QUESTION 1}

What differentials would account for this skin plaque?

\section{ANSWER 1}

The most likely diagnoses are morphoea or extragenital lichen sclerosus. Other causes of localised hypopigmentation such as vitiligo, post-inflammatory hypopigmentation, pityriasis versicolor, naevus depigmentosus and naevus anaemicus could be readily excluded on historical and clinical grounds given the induration and recent onset.

\begin{abstract}
A hypopigmented scar was considered unlikely in the absence of a convincing history of significant trauma.
\end{abstract}

\section{CASE CONTINUED}

The patient consented to having her case details and a photograph submitted to a store-and-forward teledermatology service. The dermatologist considered extragenital lichen sclerosus or morphoea to be the likely diagnosis and advised the GP to perform a punch biopsy. A $3 \mathrm{~mm}$ punch biopsy was taken from a border of the skin plaque, and histopathology reported dermal sclerosis with thickening of collagen fibres extending into the deep dermis, with focal interstitial lymphocytic infiltrate consistent with morphoea (Figure 2).

\section{QUESTION 2}

What is morphoea and how is it diagnosed?

\section{QUESTION 3}

What treatments are available?

\section{QUESTION 4}

Is there a relationship between morphoea and lichen sclerosus?

\section{QUESTION 5}

What is teledermatology?

\section{ANSWER 2}

Morphoea is a rare idiopathic condition that causes pathological skin hardening. It is also known as localised scleroderma, but this term is not recommended as it may cause confusion with limited or diffuse scleroderma. Present evidence does not suggest that patients with morphoea are at heightened risk of developing limited or diffuse scleroderma. ${ }^{1}$ Morphoea is at least twice more common in women than men. ${ }^{2}$ It typically presents with single or multiple plaques of variable depth that undergo inflammation, sclerosis and finally atrophy over several years. Morphoea can develop after external triggers such as friction or radiotherapy. ${ }^{3}$ It can cause both cosmetic and functional deficits depending on its location. Biopsy is not necessary for diagnosis. It was performed in this case to differentiate from extragenital lichen sclerosus and to exclude cutaneous breast cancer. Cutaneous breast cancer can be confused with other breast conditions so a biopsy is considered mandatory in this location. ${ }^{4}$

\section{ANSWER 3}

Treatment is not always required and should be tailored to the patient. Options include topical therapies, intralesional steroid injections, phototherapy and systemic therapies such as corticosteroids and methotrexate. Systemic agents are reserved for patients with progressive, deep or widespread morphoea. Examples of topical therapies presently used include corticosteroids, tacrolimus, calcipotriol and imiquimod. Surgery and physical therapies may be required if there are functional impairments. Treatment started early in the inflammatory phase likely improves the outcome. ${ }^{5}$ 


\section{ANSWER 4}

The relationship between morphoea and lichen sclerosus has been debated, with postulation that they share a common pathogenesis and disease process. ${ }^{6}$ There is a high incidence of lichen sclerosus in patients with morphoea. ${ }^{7}$ They are both associated with autoantibodies and autoimmune diseases. ${ }^{8}$

\section{ANSWER 5}

Teledermatology refers to the use of information technology to communicate patient details with a dermatologist. It is valuable to Australians with significant

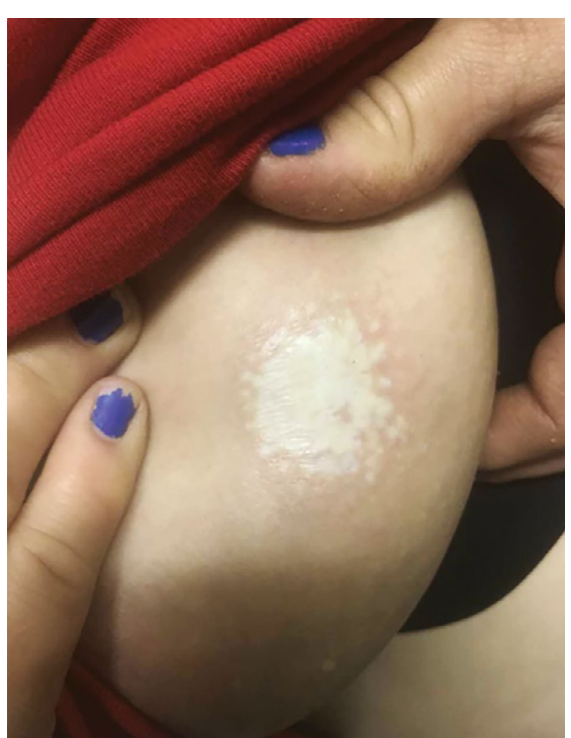

Figure 1. The skin plaque geographic separation from their nearest dermatology service and can reduce the need for face-to-face consultation. ${ }^{9}$ The two teledermatology modalities used are store-and-forward and live interaction. The store-and-forward modality is considered cost effective and useful for gaining rapid feedback and triage. ${ }^{10} \mathrm{It}$ typically involves a clinician submitting patient narrative and photographs to an online platform for review and feedback from a dermatologist. This is efficient as cases can be submitted and answered at the convenience of the involved clinicians with no requirement for the patient to be present, which is not the case for live interaction. The time taken to prepare and submit this case for advice was 15 minutes. The dermatologist response was received within 24 hours. A significant disincentive to the use of store-andforward teledermatology may be the absence of any item number under the Medicare Benefits Schedule for the doctors involved. This article gives an example of using teledermatology to diagnose and initiate treatment for an important and uncommon skin condition.

\section{CASE CONTINUED}

This result was the subject of further correspondence with the dermatologist, who suggested a management plan. The patient was recalled by the GP and assessed as having no signs or symptoms of limited or diffuse scleroderma. Blood testing was negative for antinuclear antibodies, extractable nuclear antigens, anti-DNA and RNA polymerase

III antibodies. She was prescribed mometasone furoate ointment $0.1 \%$ twice daily topically under occlusion for three weeks. At review there was minor fading and reduction of induration. Having been educated about the natural history of morphoea, the patient was happy not to pursue any further intervention. Two other small plaques subsequently developed on her breasts but spontaneously resolved.

\section{Key points}

- Morphoea can be diagnosed on the basis of typical history and appearance. A biopsy is useful if there is uncertainty.

- There is a high incidence of lichen sclerosus in patients with morphoea.

- Teledermatology is valuable to patients and GPs who may not otherwise be able to readily access a dermatologist.

- Teledermatology can be used to assess and manage challenging cases.

\section{Authors}

John O'Bryen BSc, MBBS, FRACGP,

General Practitioner, Toowoomba, Qld. john.obryen@uqconnect.edu.au

James Muir MBBS, FACD, FACRRM (Hon), Director of the Department of Dermatology, Mater Misericordiae Hospital, Qld; Dermatologist, Australian College of Rural and Remote Medicine Tele-Derm, Qld

Competing interests: None.

Funding: None.

Provenance and peer review: Not commissioned, externally peer reviewed.
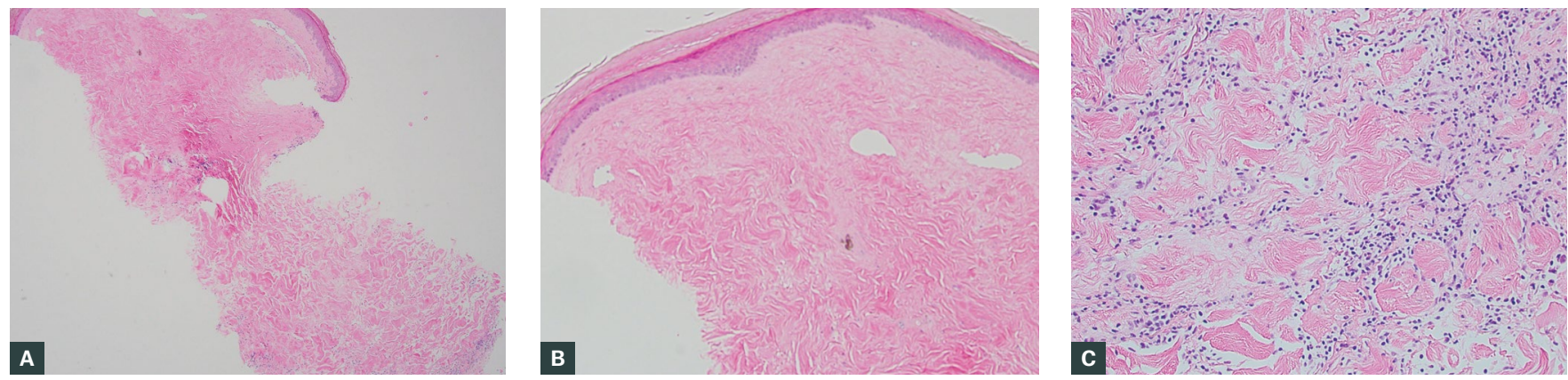

Figure 2. Histopathology images of the punch biopsy specimen

A. A sclerotic dermis and classic 'straight' biopsy sides; B. An atrophic epidermis and thickened collagen fibres with reduced interstitial space; C. Interstitial lymphocytic infiltrate focused at the base of the specimen 


\section{Acknowledgement}

Dr Rhonda Edwards, Dermatopathologist, Sullivan Nicolaides Pathology, QId, provided the histopathology images and commentary.

\section{References}

1. Lipsker D, Bessis D, Cosnes A, et al. Prospective evaluation of frequency of signs of systemic sclerosis in 76 patients with morphea. Clin Exp Rheumatol 2015;33(4 Suppl 91):S23-25.

2. Peterson LS, Nelson AM, Su WP, Mason T, O'Fallon WM, Gabriel SE. The epidemiology of morphea (localized scleroderma) in Olmsted County 1960-1993. J Rheumatol 1997;24(1):73-80.

3. Vigneron C, Bauer N, Waisse W, et al. Radio-induced breast morphoea: An unrecognized complication. Cancer Radiother 2014 Jan;18(1):64-67. doi: 10.1016/j. canrad.2013.09.006.

4. Apel W, Wilson K, Anderson D. Atypical dermatology - Are you missing an important diagnosis? Aust Fam Physician 2014;43(7):451-53.

5. Zwischenberger BA, Jacobe HT. A systemic review of morphea treatments and therapeutic algorithm. J Am Acad Dermatol 2011;65(5):925-41. doi: 10.1016/j.jaad.2010.09.006.

6. Farrell AM, Marren PM, Wojnarowska F. Genital lichen sclerosus associated with morphoea or systemic sclerosis: Clinical and HLA characteristics. Br J Dermatol 2000;143(3):598-603. doi: 10.1111/j.1365-2133.2000.03717.x.

7. Lutz V, Francès $C$, Bessis D, et al. High frequency of genital lichen sclerosus in a prospective series of 76 patients with morphea: Toward a better understanding of the spectrum of morphea. Arch Dermatol 2012;148(1):24-28. doi: 10.1001/ archdermatol.2011.305.

8. Leitenberger JJ, Cayce RL, Haley RW, Adams-Huet B, Bergstresser PR, Jacobe HT. Distinct autoimmune syndromes in morphea: A review of 245 adult and pediatric cases. Arch Dermatol 2009;145(5):545-50. doi: 10.1001/ archdermatol.2009.79.

9. Lee KJ, Finnane A, Soyer HP. Recent trends in teledermatology and teledermoscopy. Dermatol Pract Concept 2018;8(3);214-23. doi: 10.5826/ dpc.0803a13.

10. Snoswell C, Finnane A, Janda M, Soyer HP, Whitty JA. Cost-effectiveness of Store-andForward teledermatology: A systemic review. JAMA Dermatol 2016;152(6):702-08. doi: 10.1001/ jamadermatol.2016.0525. 